\title{
Measurements with the stripping foil test stand in the Linac4 Transfer Line
}

\author{
Chiara Bracco ${ }^{1}$, Louise Jorat ${ }^{1}$, Remy Noulibos $^{1}$, Pieter van Trappen ${ }^{1}$, and Wim Weterings ${ }^{1, *}$ \\ ${ }^{1}$ CERN, CH-1211, Geneva 23, Switzerland
}

\begin{abstract}
In 2020, after the CERN accelerators complex Long Shutdown 2 (LS2), a novel Linac4 (L4)-toPS Booster (PSB) charge-exchange injection system will allow to transform the $\mathrm{L} 4160 \mathrm{MeV} \mathrm{H}^{-}$beam into $\mathrm{H}^{+}$, which will be injected into the four PSB superposed rings. For this, a $200 \mu \mathrm{g} / \mathrm{cm}^{2}$ carbon stripping foil will convert negative hydrogen ions $\left(\mathrm{H}^{-}\right)$into protons by stripping off the electrons. L4 is now performing operational reliability runs, which include a stripping foil test stand installed in the L4 transfer line. These tests will permit to gain experience on the fragile foils, test different foil materials and thicknesses, measure the efficiency and lifetime of the foils, and evaluate the foil changing mechanism as well as the interlocking functions. This paper briefly describes the stripping foil test stand setup, before reporting on the obtained important test results.
\end{abstract}

\section{Introduction}

At CERN, the European Organization for Nuclear Research, particle accelerators and detectors are used to study the basic constituents of matter. Since its foundation in 1954, physicists and engineers have unified effort to increasing the discovery potential and pushing the limits of the energy frontier. The flagship accelerator of CERN is the Large Hadron Collider (LHC), world famous for being the most powerful particle accelerator ever built.

A massive improvement program of the LHC injector chain is presently being conducted under the LHC Injectors Upgrade (LIU) project [1] with the aim of producing the challenging High Luminosity LHC (HL-LHC) beam parameters [2]. The project comprises a new Linac, socalled Linac4 (L4), as well as major upgrades and consolidation of the Proton Synchrotron Booster (PSB), the Proton Synchrotron (PS) and the Super Proton Synchrotron (SPS).

L4 is a linear accelerator [3] intended to deliver a beam at $160 \mathrm{MeV}$ energy to the four superposed synchrotron rings of the PSB. The main improvements of $\mathrm{L} 4$ stem from the use of negative hydrogen ions $\left(\mathrm{H}^{-}\right)$instead of protons $\left(\mathrm{H}^{+}\right)$with a higher injection energy than the currently used $50 \mathrm{MeV}$ of Linac2 and the PSB will be equipped with an $\mathrm{H}^{-}$charge-exchange injection system during the Long Shutdown 2019-2020 (LS2). Following the upgrade, $\mathrm{H}^{-}$ will be progressively injected horizontally into the PSB and converted into $\mathrm{H}^{+}$by passing through a $200 \mu \mathrm{g} / \mathrm{cm}^{2}$ carbon foil to strip them of the electrons, aiming to convert $\sim 98 \%$ of the $\mathrm{H}^{-}$beam to $\mathrm{H}^{+}$[4]. Partially stripped $\mathrm{H}^{0}$ and $\sim 1 \% \mathrm{H}^{-}$missing the foil will be directed to an internal $\mathrm{H}^{0} / \mathrm{H}^{-}$dump located inside an injection chicane magnet [5]. This charge-exchange injection technique can achieve higher particle density providing an extremely

\footnotetext{
*Corresponding author: wim.weterings@cern.ch
}

flexible way to load particles into the PSB, making the accumulation of many turns possible with a tight control of the beam density [6].

In 2016 beam commissioning of L4 took place in steps of increasing energy to reach $160 \mathrm{MeV}$ [7] and L4 is now performing operational reliability runs. A stripping foil test stand is installed in the L4 transfer line including the identical foil handling and exchange mechanism that will be used in the future PSB $\mathrm{H}^{-}$charge-exchange injection system [8]. This will allow to gain experience using these fragile foils, test different foil materials and thicknesses, evaluate the lifetime of the foils and foil holders and test the foil changing mechanism as well as the interlocking functions prior to the final installation in the PSB in 2019.

\section{Stripping foil test stand}

The design of the stripping foil handling and exchange mechanism, so-called TKSTR, of which an illustration is given in Figure 1, was extensively presented in [8, 9]. Based on commercially available components [10], the unit for the L4 test stand is identical as the mechanism intended to be installed in the PSB. It consists of a stainless steel belt, rotating over two pulleys, to which a maximum of six foil holders can be attached by use of quick disconnect sliders. This allows moving a foil into the beam aperture, with a perpetual rotation, so that each of the six foils can be re-selected into the nominal beam position with a precision of $\pm 0.1 \mathrm{~mm}$ from which a foil movement in the horizontal plane of $\pm 2 \mathrm{~mm}$ is possible in order to find the optimum position for operation. To this extend the mechanism is equipped with UHV compatible microswitches and membrane potentiometers, a detailed description of the electronics and controls for the TKSTR was presented in [11]. 


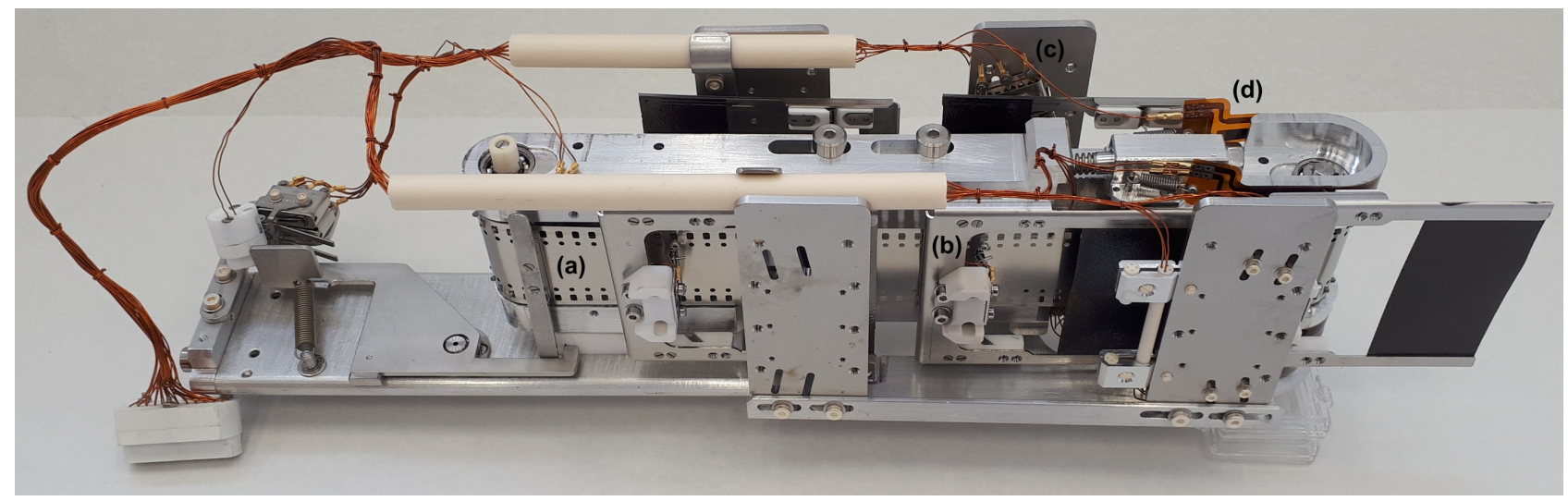

Fig. 1. The stripping foil exchange mechanism showing the rotating stainless steel belt (a) [10], the holders with stripping foils attached (b), the UHV compatible microswitches (c) and the membrane potentiometers (d) for the electronics and controls [11].

\section{Test conditions}

\subsection{Beam characteristics}

According to the latest specifications, L 4 is s upposed to reach a peak current of $40 \mathrm{~mA}$. During nominal operation, some bunches will have to be chopped away to avoid losses during the transfer to the four superposed rings and during the longitudinal capture in the PSB. Finally, a peak current of $26 \mathrm{~mA}$ is expected at the PSB after chopping. Four pulses, one per ring, of up to $150 \mu \mathrm{s}$, will have to be produced with a flatness in current of $\pm 5 \%$.

All the foils at the test stand were tested with $160 \mathrm{MeV} \mathrm{H}^{-}$beam with a transverse r.m.s. emittance of $0.4 \pi \cdot \mu \mathrm{m} \cdot \mathrm{mrad}$ mainly defined by the a cceptance of the RFQ. Presently, a current of 30-35 mA has been reached in the linac and up to $20 \mathrm{~mA}$ could be transported to the foil. During the first dry run, in S pring 2018, the current at the foil was slowly increased from $5 \mathrm{~mA}$ to $20 \mathrm{~mA}$ and the pulse length from $200 \mu$ s to $600 \mu$ s. Initially, large current variations were observed along the pulse, when operating with $600 \mu \mathrm{s}$. Several optimizations were applied to the linac and the transfer line and a $\pm 5 \%$ flatness could finally be achieved over the full pulse length. The quality of the stripping efficiency measurements over the Spring and Autumn Dry Runs reflect these more stable conditions and a clear reduction in the measured standard deviation was found.

In normal operation, in the PSB, each foil will see only pulses of maximum $150 \mu$ s but multiple crossings have to be considered, at slightly different locations of the foil due to the transverse painting, for the circulating beam. The beam conditions used during the tests, as summarized in Table1, allow to evaluate both the stripping efficiency and extrapolate the lifetime of each foil type during standard operation.

\subsection{Stripping foils}

The stripping foils that were used for the beam tests are commercially available and the characteristics are shown in Table 2. The required foil thickness for PSB injection
Table 1. Characteristics of the beam used for the tests.

\begin{tabular}{cll}
\hline Parameters & Value & Unit \\
\hline Ion Species & $\mathrm{H}^{-}$ & \\
Energy & 160 & $\mathrm{MeV}$ \\
Repetition Rate & 1.2 & $\mathrm{~s}$ \\
Pulse Length* & $200-600$ & $\mu \mathrm{s}$ \\
Mean pulse Current & $5-20$ & $\mathrm{~mA}$ \\
Transverse emittance & 0.4 & $\pi \cdot \mu \mathrm{m} \cdot \mathrm{mrad}$ \\
$\mathrm{H} / \mathrm{V}$ beam size @ foil & $\sim 2.3 / 2.9$ & $\mathrm{~mm}(\mathrm{radius})$ \\
\hline
\end{tabular}

$200 \mu$ s to $600 \mu$ s pulses with $50 \mu$ s to $150 \mu$ s batches

is $200 \mu \mathrm{g} / \mathrm{cm}^{2}(\sim 1 \mu \mathrm{m})$ to ensure a theoretical stripping efficiency $>99 \%$ while keeping the emittance increase below $\sim 0.1 \mathrm{~mm}$ mrad for the LHC beam (required $\sim 2 \mathrm{~mm}$ mrad rms normalised emittance at injection) and the uncontrolled beam losses below the $10^{-4}$ level [12].

The $32 \mathrm{~mm}$ wide foils are glued, on identical $58 \mathrm{~mm}$ vertical beam aperture stainless steel frames, with a mixture of $50 \%$ demineralised water and $50 \%$ Aquadag ${ }^{\circledR} 18 \%$ solution, of which a detailed description is presented in [9]. An illustration of the stripping foil holder attached to the rotating belt is given in Figure 2 .

\subsection{Measurements with BTC}

Two beam current transformers (BCT), located upstream (L4T) and downstream (L4Z) of the foil, are used to measure the stripping efficiency by looking at their relative current decrease. Following the issues of a $20 \%$ higher signal at the downstream monitor, reported in [9], a cross-calibration of these BCTs at the percent level was done. The observed preamplifier saturation effects, due to a strong $352 \mathrm{MHz}$ RF component well outside the presumed passband of the transformer, was corrected by the addition of appropriate low pass filters to have a reliable evaluation of the number of protons with respect to the original injected $\mathrm{H}^{-}$[18]. Figure 3 shows a screen shot of the BCT control interface for operation and acquisition. The BCT positions L4T and L4Z respectively are the upstream and downstream BCTs with respect to the TKSTR. 
Table 2. Characteristics of the different foil types used.

\begin{tabular}{cllll}
\hline \multicolumn{1}{c}{ Description } & Weight & Remark & Name & \\
\hline Arc evaporated amorphous carbon & $200 \mu \mathrm{g} / \mathrm{cm}^{2}$ & Collodion coated & XCF-200 & {$[13]$} \\
Arc evaporated amorphous carbon & $199 \mu \mathrm{g} / \mathrm{cm}^{2}$ & & GSI-199 & {$[14]$} \\
Diamond-like carbon & $200 \mu \mathrm{g} / \mathrm{cm}^{2}$ & Boron doped 10\% & DLC-23-1000-S & {$[15]$} \\
Multilayer graphene & $200 \mu \mathrm{g} / \mathrm{cm}^{2}$ & & MLG-200 & {$[16,17]$} \\
Multilayer graphene & $233 \mu \mathrm{g} / \mathrm{cm}^{2}$ & & MLG-233 & {$[16,17]$} \\
Multilayer graphene & $251 \mu \mathrm{g} / \mathrm{cm}^{2}$ & & MLG-251 & {$[16,17]$} \\
\hline
\end{tabular}

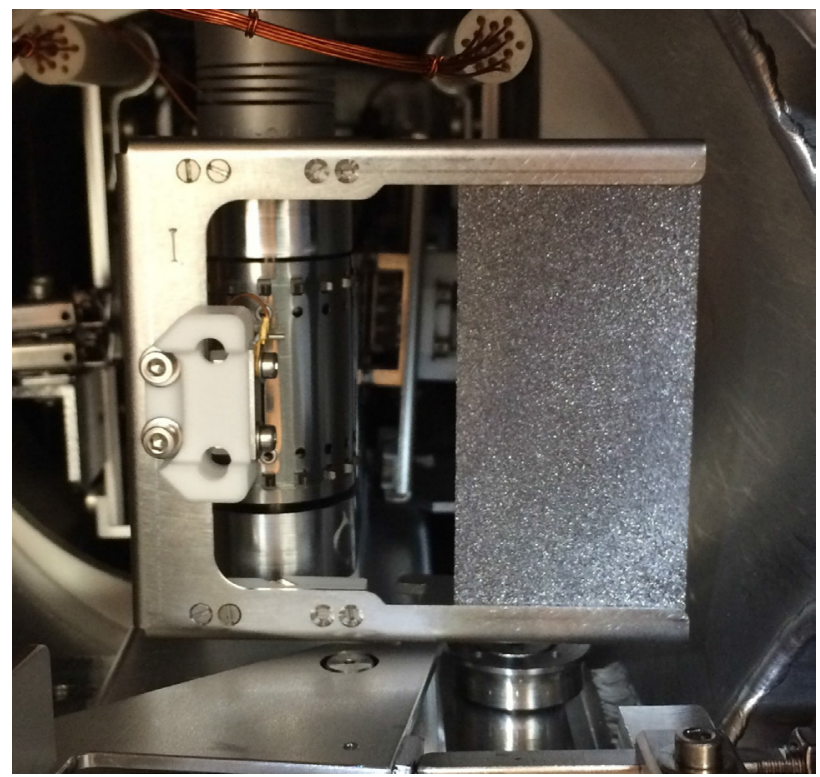

Fig. 2. A MLG-251 stripping foil mounted on the stainless steel foil holder, attached to the rotating belt by use of a quick disconnect slider. The foil is $32 \mathrm{~mm}$ wide and the vertical beam aperture of the holder is $58 \mathrm{~mm}$.

A focusing quadrupole (L4T.RQF.021) for the $\mathrm{H}^{-}$ions optics is installed after the stripping foil test stand. During the stripping foil tests, this quadrupole has to be switched off to maximize the transport of the protons when the foil is intercepting the beam and the status of charge is inverted. A clear effect on the measured stripping efficiency could be seen when the quadrupole was accidentally left on (see Figure 5).

\subsection{Setting up with BTV}

Correct position of the foil, with respect to the beam, is important for optimizing the stripping efficiency and for this reason a retractable optical beam observation system (BTV), consisting of a $1 \mathrm{~mm}$ thick Chromox $\left(\mathrm{Al}_{2} \mathrm{O}_{3}\right.$ doped with $\mathrm{CrO}_{2}$ ) scintillating screen, can be placed $6 \mathrm{~mm}$ in front of the foil [19]. The image of the screen, or of the stripping foil, is recorded by a radiation-hard camera, allowing checking either the beam position or the integrity of the foil [9]. Before measuring the stripping efficiency of a foil, a single beam pulse is shot on the BTV screen, to determine its position. Subsequently the foil edge can be moved $\pm 2 \mathrm{~mm}$, or the beam optics adjusted, in order

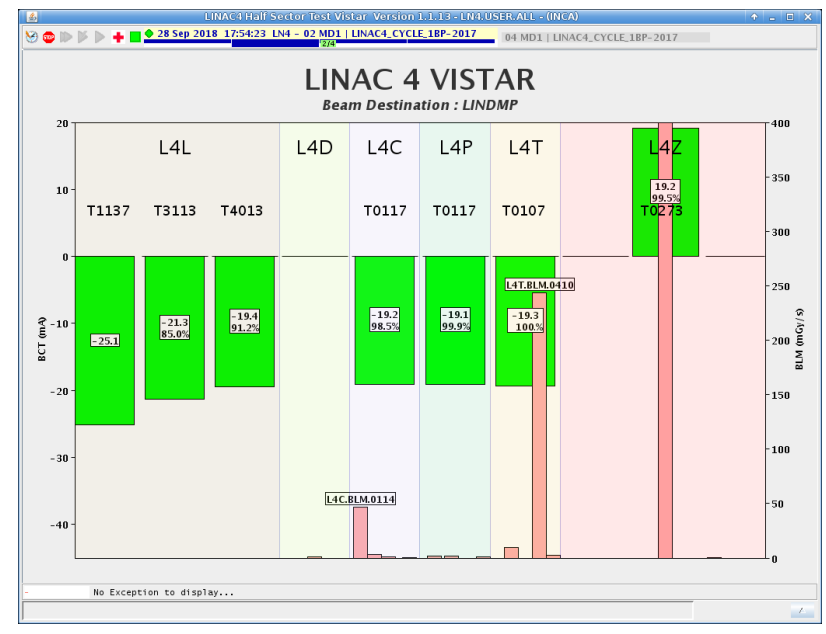

Fig. 3. Screen shot of the BCT control interface for operation and acquisition showing the beam current at different BCT positions in the L4. Location L4T (-19.3mA) and L4Z (19.2mA) respectively are the upstream and downstream BCTs with respect to the TKSTR. The readings of the Beam Loss Monitors (BLMs) are also shown.

to achieve the theoretical stripping point position of the beam, which is at $14 \mathrm{~mm}$ from the edge of the foil.

During the first beam tests, that took place in October 2016, two foils (XCF-200) got broken while continues beam pulses were sent on the BTV during $\sim 1$ minute periods. After removing the screen, the foil reappeared damaged [9]. The mechanism that caused this foil disruption was thought to be the beam induced charging of the screen, and probably also the foil, during beam impacts causing rupture when the screen is moved. Since the improvement of the electrical contacts of the foil frames and the application of a silver conducting layer on the backside of the BTV screen this phenomena has not anymore been observed.

Another catastrophic failure occurred in September 2018. While setting up for tests with beam the BTV screen horizontally broke in the middle, see Figure 4. It is possible that continues beam shots on the screen, done to investigate the influence of the improved electrical contact and coating of the screen described above, have stressed the screen causing breakage. During a subsequent technical stop, inspection through the viewport showed the top part of the screen broken in two part at the bottom of the vacuum tank. 


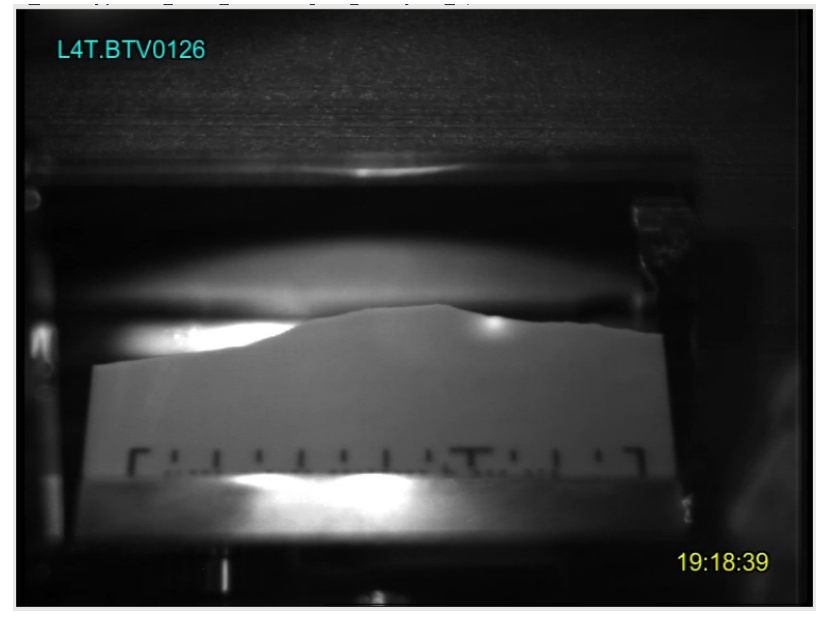

Fig. 4. Image from the radiation-hard camera of the broken BTV screen. The foil holder, ready to be put into beam position, can be seen at the right behind the screen. The glow on the top edge, right from the centre of the screen, is the position of beam impact previous to breakage.

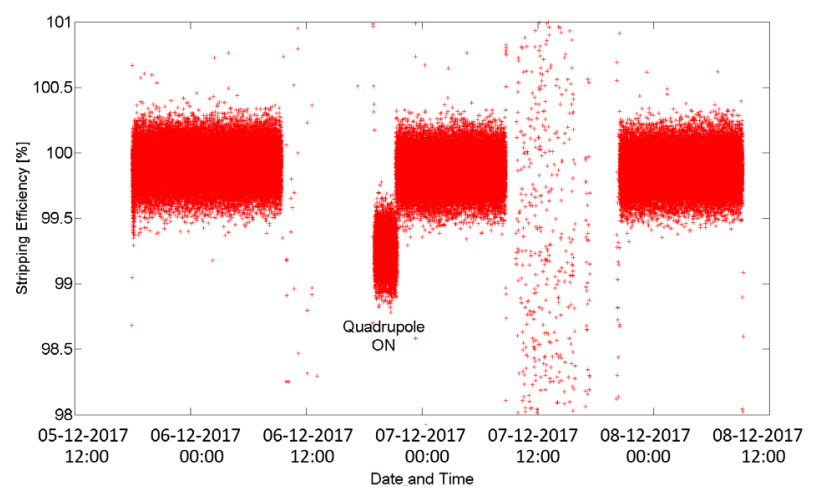

Fig. 5. Efficiency measurement data, from 3 night-shift runs, with $400 \mu$ s pulses of $\sim 15.7 \mathrm{~mA}$ beam current. The influence of incorrect beam optics, L4T.RQF.021 quadrupole on, can clearly be identified.

\section{Test results}

\subsection{Stripping efficiency measurements}

Throughout the L4 reliability run, stripping foil efficiency measurements took place during night-shifts and weekends, the day shift being dedicated to machine development and beam diagnostics. During the measurements, all L4T and L4Z BCT data was logged and post-processed to determine the stripping efficiency, Figure 5 shows the collected data of a typical run.

Taking into account data from several runs, and discarding all measurements outside the $98-100 \%$ data range, the stripping efficiency of the foils mentioned in Table 2 was calculated and is presented in Figure 6. One can observe that the efficiency of all foils is well above the theoretical value of $>99 \%$, as described in section 3.2, with the exception of MLG-200. According to experts, the observed behavior in terms of stripping efficiency and losses is as expected for multi-layer graphene foils [16]. Despite

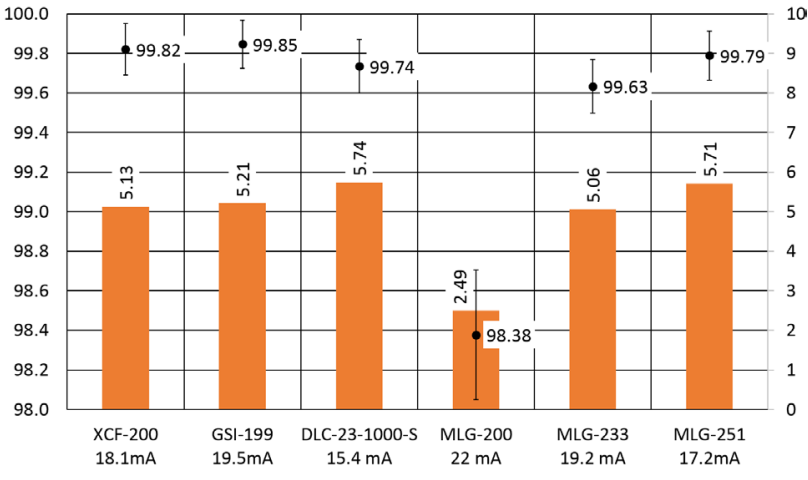

Fig. 6. Stripping efficiency measurements of the foils in the L4 test stand with $600 \mu$ s beam pulses and indicated beam current. The left axis shows the efficiency [\%] of the foils and the right axis the beam losses $[\mathrm{mGy} / \mathrm{s}]$.

the same thickness of $200 \mu \mathrm{g} / \mathrm{cm}^{2}$, as the other carbonbased foils, the crystalline structure of the graphene reduces the probability of interaction with the $\mathrm{H}^{-}$ions and thus the charge-exchange process. On the other hand, the reduction of the cross sections for nuclear interaction, single and multi-Coulomb scattering determines the favorable effect of decreasing both the losses and the emittance blow up. For all these reasons, and for the interesting mechanical properties which make these foils relatively easy to handle, it was decided to test thicker graphene foils (MLG-233 and MLG-251) and assess their stripping efficiency. Unfortunately the emittance blowup induced by the different foils cannot be measured with the present installation since the effect of a single passage across the foil is below the resolution of the available diagnostics. Still the characterization of the foils in terms of losses and efficiency will provide useful information for bench-marking with simulations which will then allow to predict the expected effect on the final emittance in the PSB.

\subsection{Foil damage and observations}

Movement at the beam spot location of the DLC-23-1000$\mathrm{S}$ and XCF-200 foils is observed during every beam shot, very well distinguished at the frequency of the L4 beam pulse. Inspection of the tested foils during a technical stop showed clear deformation and the creation of a crater, as shown in Figure 7, at impact location of foil DLC-231000-S. Nevertheless, subsequent tests showed that this phenomena has no influence on the stripping efficiency of the foil.

During tests, taking place in March 2018, it was observed that a DLC-23-1000-S foil, which had not been in beam position, was completely removed from the foil holder. Following inspection during the next technical stop, it was found lying inside the vacuum tank and looked completely burnt. Also delamination of foil layers was noticed at the edge of the foil, as shown in Figure 8, and the foil holder of this foil was particularly activated, $>38 \mu \mathrm{Sv}$ at contact, compared to $0.1 \mu \mathrm{Sv}$ of all other frames. High losses at the stripping foil test stand and a very low transmission to the downstream BCT were recorded, on March 


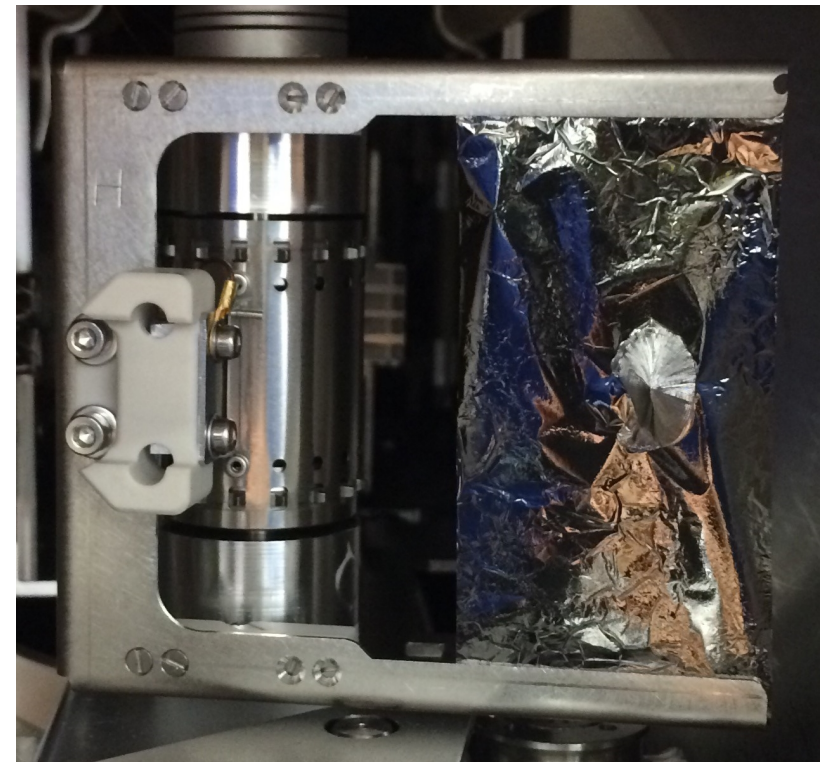

Fig. 7. Appearance of a crater like deformation on a DLC-231000-S foil. Movement at the beam spot location is observed during every beam shot.

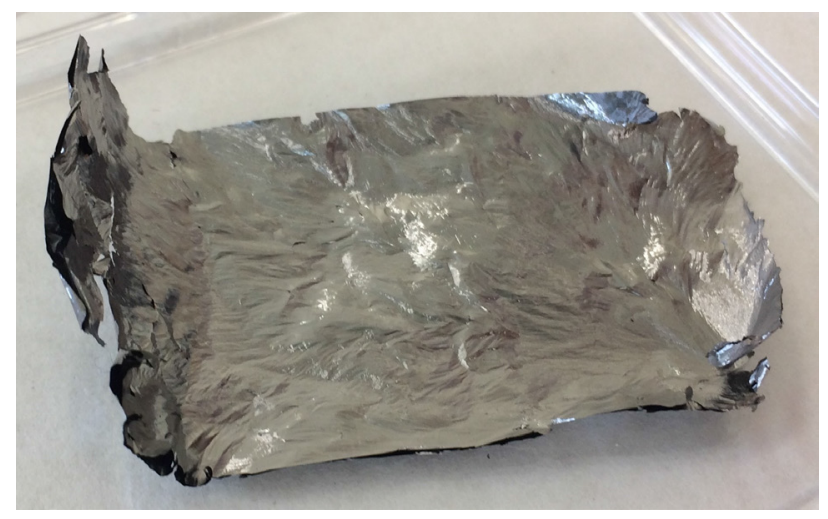

Fig. 8. DLC-23-1000-S foil that was detached from the foil without being tested with beam. The hypotheses is that the frame of this foil was hit by the showers of particles produced by missteered beam hitting the machine aperture, just upstream of the loader, during low energy tuning of the linac.

$15^{\text {th }}$, during the low energy tuning of the linac cavities at $3 \mathrm{MeV}$ as shown in Figure 9. Neither the foil or the BTV were intercepting the beam at that moment so the most likely source of these losses was a mis-steered beam hitting the machine aperture upstream of the test stand. The frame holding DLC-23-1000-S foil was facing the beam direction when the losses occurred and it might thus be fully hit by the electromagnetic secondary showers. This hypotheses was confirmed by damage that occured, due to irradiation, to the downstream electronics.

In October 2018, the GSI-199 type was tested for the first time. The foil was moved in beam position and after only one beam pulse the aspect of the foil completely changed, as shown in Figure 10, it looked like the foil shrunk or deformed towards the center of beam impact. Nevertheless, this had no influence on the performance of

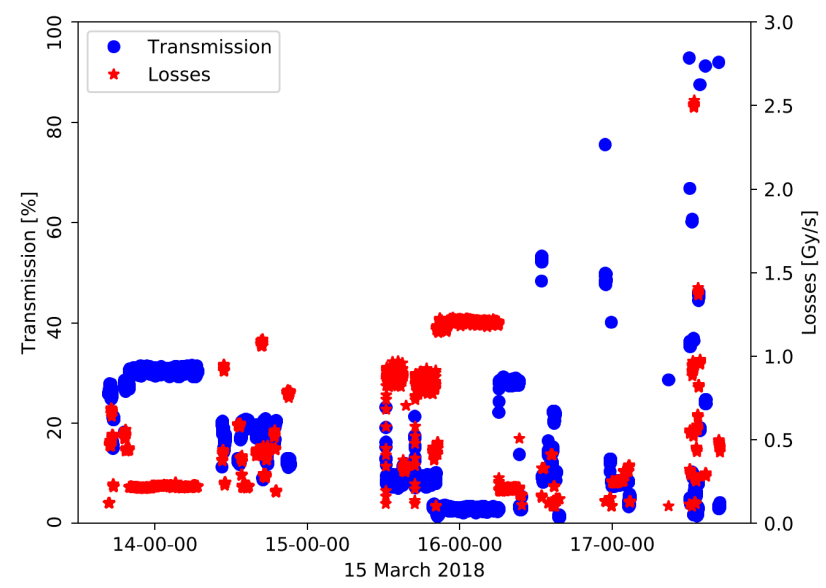

Fig. 9. Losses and current transmission during $3 \mathrm{MeV}$ low energy tuning of the linac on March $15^{\text {th }}$. The ratio between the current of the upstream and downstream BCT values are shown in blue and the losses at the BLM located just in front of the stripping foil unit are shown in red.

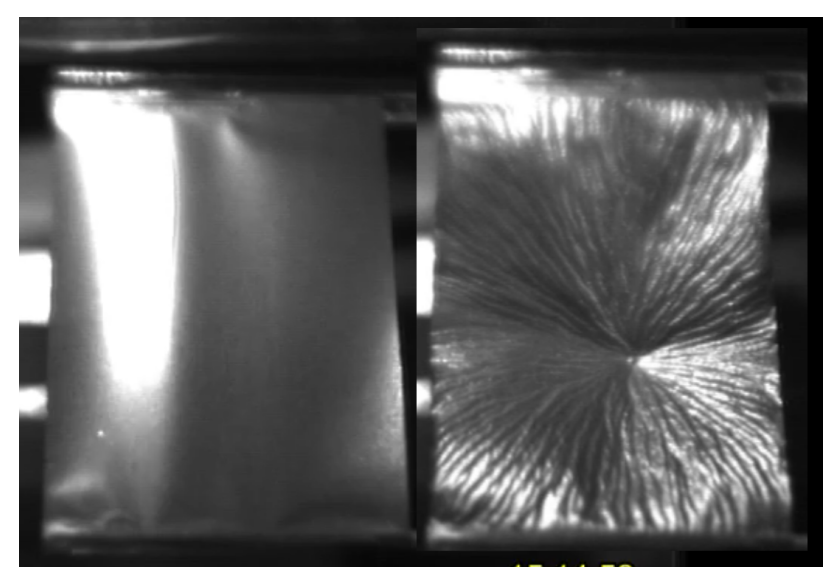

Fig. 10. GSI-199 foil before (left) and after (right) one $600 \mu \mathrm{s}$ pulse of $\sim 19.5 \mathrm{~mA}$. This effect had no influence on the stripping efficiency of the foil.

the foil, it remained in beam for 9 hours with a stripping efficiency of $\sim 99.8 \%$.

At another occasion, a foil frame got stuck behind a microswitch, but the system could be unblocked by reversing the movement of the stainless steel band. Nevertheless, this manoeuvre caused rupture of the DLC-23-1000-S foil.

\section{Conclusion}

A stripping foil test stand is installed in the L4 transfer line, including the identical foil handling and exchange mechanism that will be used in the new $\mathrm{H}^{-}$charge exchange injection system of the PSB when L4 is connected with $160 \mathrm{MeV}$ injection energy. During the L4 operational reliability runs the behaviour under beam conditions of several types of foils has been evaluated with measurement of their stripping efficiency using calibrated BCTs. All tested foil types have the expected theoretical stripping efficiency $>99 \%$. 
Movement at the beam spot on foils is observed during every beam shot, very well distinguished at the frequency of the L4 beam pulse, and some foils show clear deformation at the impact location. This phenomena has no influence on the stripping efficiency of the foils.

Damage and increased radiation of a foil, which had not been in beam position, was observed and this phenomenon is thought to be caused by uncontrolled losses during the low energy tuning at $3 \mathrm{MeV}$ of the $\mathrm{L} 4$ beam impacting on the stripping foil mechanism.

The authors would like to acknowledge the help and support of the CERN Control Center (CCC) operators during the stripping foil efficiency tests.

\section{References}

1. E. Shaposhnikova et al., LHC Injectors Upgrade (LIU) Project at CERN, in Proceedings, 7th International Particle Accelerator Conference (IPAC 2016): Busan, Korea, May 8-13 (2016), p. MOPOY059

2. G. Apollinari, O. Brüning, L. Rossi, High Luminosity LHC Project Description, in Report (2014), CERNACC-2014-0321

3. F. Gerigk, M. Vretenaar et al., Linac 4 Technical Design Report, in Report (2006), CERN-AB-2006-084

4. E. Benedetto et al., Status of the $160 \mathrm{MeV} \mathrm{H}^{-}$Injection into the CERN PSB, in Proceedings, 3rd International Conference on Particle accelerator (IPAC 2012): New Orleans, USA, May 20-25 (2012), p. TUPPR091

5. M. Delonca, C. Maglioni, A. Patapenka, A.S. Martinez, Internal $\mathrm{H}^{0} / \mathrm{H}^{-}$Dump for the Proton Synchrotron Booster Injection at CERN, in Proceedings, 3rd International Conference on Particle accelerator (IPAC 2012): New Orleans, USA, May 20-25 (2012), p. TUPPR054

6. J.L. Abelleira, W. Bartmann, E. Benedetto, C. Bracco, G.P. Di Giovanni, V. Forte, M. Kowalska, M. Meddahi, B. Mikulec, G. Rumolo, Painting Schemes for CERN PS Booster $H^{-}$Injection, in Proceedings, 6th International Particle Accelerator Conference (IPAC 2015): Richmond, Virginia, USA, May 3-8 (2015), p. THPF083
7. A. Lombardi, Linac4: From Initial Design to Final Commissioning, in Proceedings, 8th International Particle Accelerator Conference (IPAC 2017): Copenhagen, Denmark, May 14-19 (2017), p. TUYA1

8. W. Weterings, C. Bracco, R. Noulibos, Y. Sillanoli, P. van Trappen, J Radioanal Nucl Chem 305, 831 (2015)

9. W. Weterings, C. Bracco, L. Jorat, R. Noulibos, P. van Trappen, AIP Conference Proceedings 1962, 030003 (2018)

10. NEC, 7540 Graber Road, P.O. Box 620310, Middleton, Wisconsin 53562

11. P. Van Trappen, R. Noulibos, W. Weterings, Stripping Foil Displacement Unit Control for $\mathrm{H}^{-}$Injection in PSB at CERN, in Proceedings, 15th International Conference on Accelerator and Large Experimental Physics Control Systems (ICALEPCS 2015): Melbourne, Australia, October 17-23 (2015), p. MOPGF121

12. B. Goddard, M. Aiba, C. Bracco, C. Carli, M. Meddahi, W. Weterings, Stripping Foil Issues for $\mathrm{H}^{-}$Injection into the CERN PSB at $160 \mathrm{MeV}$, in Proceedings, 1st International Conference on Particle accelerator (IPAC 2010): Kyoto, Japan, May 23-28 (2010), p. THPEB030

13. ACF-Metals, 2239 E. Kleindale Road, Tucson, Arizona, U.S.A

14. GSI, Helmholtzzentrum für Schwerionenforschung GmbH, Planckstraße 1, 64291, Darmstadt, Germany

15. MICROMATTER, Unit \#1, 8333 - 130th Street, Surrey, BC V3W 7X4, Canada

16. A. Tatami, KANEKA Corporation, 5-1-1, TorikaiNishi, Settsu Osaka 566-0072, Japan

17. A. Tatami, M. Tachibana, T. Yagi, M. Murakami, AIP Conference Proceedings 1962, 030005 (2018)

18. F. Roncarolo et al., Beam Instrumentation for the CERN LINAC4 and PSB Half Sector Test, in Proceedings, 8th International Particle Accelerator Conference (IPAC 2017): Copenhagen, Denmark, May 1419 (2017), p. MOPAB120

19. S. Burger, Beam Observation System (BTV) at Stripping Foil for PSB $\mathrm{H}^{-}$Injection, in Engineering Specification 1706232 (2016), https://edms. cern.ch/ document/1706232/1 